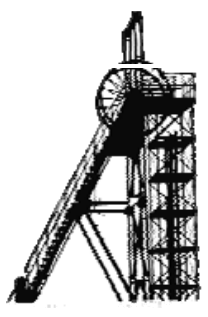

\title{
¿Es posible abordar el folklore musical de otras comunidades autónomas en el segundo ciclo de Educación Infantil? El caso del flamenco
}

\author{
Adrián Sánchez García \\ Universidad de Valladolid \\ Lidia Álava Redal \\ Graduada en Educación Infantil \\ Enviado: $13-10-2020$ \\ Aceptado: $23-12-2020$
}

\section{Resumen}

En este texto pretendemos comprobar la posibilidad de que el alumnado escolarizado en el segundo ciclo de Educación Infantil experimente, dentro del contexto escolar y en función de lo que permitan los diferentes currículos españoles, el folclore musical de otra comunidad autónoma. Para ello, analizamos la normativa vigente en este ciclo de cada comunidad autónoma en torno a la didáctica del folclore y nos centramos en la especial casuística del flamenco, declarado Patrimonio Cultural de la Humanidad de la UNESCO.

Palabras clave: flamenco, folklore musical, educación infantil, didáctica del folklore.

\section{Abstract}

In this text, we hope to verify the chance that preschool students experience, within the school context, the musical folklore of other regions, always depending on what the Spanish syllabus allow us. In order to do this, we analyze the current law in this course of every region about folklore teaching and we focus on special casuistry of flamenco, declared to be World Cultural Heritage by UNESCO. 


\section{Introducción}

Es necesario comenzar exponiendo que este artículo nace con una concepción que, probablemente, los amantes, estudiosos y defensores del flamenco no compartan con nosotros: el flamenco forma parte del folclore musical andaluz. Sin embargo, deseamos que este hecho no suponga un impedimento para continuar con su lectura, sino una muestra de sinceridad y respeto hacia éste y hacia todo el folclore musical español.

De hecho, se ha escrito bastante sobre la cuestión de si el flamenco es arte o folclore, por ello lo abordamos brevemente en este momento. Partimos de la base de que:

Andalucía es una tierra rica en folclore musical. [...] Sin embargo, la música más representativa y conocida de Andalucía es el Flamenco, un arte musical, literario y coreográfico compuesto por cuatro aspectos interrelacionados: el cante, el toque (la guitarra) y el baile, más uno muchas veces olvidado y sin embargo imprescindible, la letra o soporte poético (Cenizo, 2009, p. 19).

Así, Utrilla (2007) concibe el flamenco como la «más honda expresión artística del pueblo andaluz y es por ello que no debe ser considerado ni presentado como una manifestación de tipo folclórico. No es folclore el arte flamenco, aunque sus raíces sean populares» (p. 14).

Comprendemos lo que sienten y propugnan estos autores. No obstante, nuestra visión se encauza con la de Sánchez y Navarro (1998), quienes entienden el flamenco como el folklore resultante de la interrelación cultural de los individuos y el entorno, exhibido en el cante flamenco.

Incluso diferentes argumentos que son usados por Utrilla (2007) para defender que el flamenco es arte y no folclore, pueden ser asimismo empleados, según nuestra visión, para lo opuesto. Utrilla expone:

En primer lugar, el flamenco es creación personal e individual, ha sido y es fruto de las más diversas creaciones personales de sus protagonistas: cantaores, bailaores y tocaores 


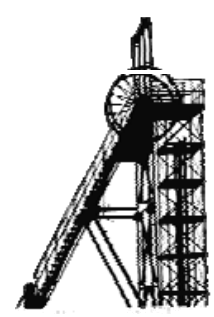

flamencos, aunque aquéllas se hayan inspirado en la música popular y folclórica, y se hayan perpetuado y transmitido en gran parte de manera oral, de padres a hijos, como ocurre en el ámbito folclórico (2007, p. 14).

Si el flamenco es una manifestación cultural que, pese a ser de creación personal, emana de la música popular de carácter folclórico, en nuestra opinión sigue siendo música popular o folclórica, puesto que al pertenecer al pueblo evoluciona con él y con los tiempos. Del mismo modo, Utrilla (2007) argumenta que esta evolución contrasta con «el inmovilismo conservador y tradicionalista que caracteriza al folclore» (p. 15). Sin embargo, consideramos que ambos, la tradición conservadora y la evolución e innovación, forman parte de una misma rama: el folclore.

Además, «su carácter y dimensión universal lo convierten en una manifestación musical cotizada y admirada en todo el mundo, mientras que el folclore tiene un carácter más regional o localista» (Utrilla, 2007: 14). Así, al flamenco también le afecta la globalización que vivimos y la hibridación o fusión con otras músicas. En este caso, sí que creemos que debería existir el debate de si es considerado folclore o no. Ahora bien, ¿no puede ser un folclore musical aceptado, querido e interpretado por otros y en otros lugares del mundo? ¿Acaso el gusto por un determinado folclore solo es posible si se tienen raíces en esa tierra? De hecho, «la copla es universal, válida para todos y no sólo algo para andaluces o para aficionados» (Cenizo, 2009, p. 33).

También Utrilla (2007) manifiesta que:

Su gran calidad, complejidad musical y dificultad técnica le confieren una magnitud artística y hacen necesaria, sin ir más allá, una formación amplia y prolongada en el tiempo, lo que marca una diferencia con el folclore más llano y popular (p. 14).

Quizá este sea el punto en el que más de acuerdo estemos. Sin embargo, además del flamenco que podríamos denominar "sofisticado", también encontramos un flamenco más asequible, con sucesiones de acordes no tan 
elaboradas, con una dedicación vocal menos exigente o con unos pasos de baile más sencillos para el público en general. Ambos flamencos constituyen un corpus que podemos clasificar por dificultad, pero, pese a necesitarse más o menos formación, sigue siendo folclore. Tampoco debemos perder de vista que, a veces, lo sencillo es más bello e interesante que lo complejo y enrevesado, debido a su poder de transmitir, evocar y emocionar desde la sencillez y naturalidad.

Dejando este tema a un lado, en el actual transcurrir del primer cuarto del siglo XXI ya conocemos la importancia de la introducción del folclore en las aulas de Educación Infantil, pero ese no es el principal objetivo de este trabajo. En las páginas que siguen intentaremos comprobar la posibilidad de que el alumnado escolarizado en el segundo ciclo de esta etapa experimente, dentro del contexto escolar, el folclore musical de otra comunidad autónoma, según las diversas normativas vigentes en nuestro país. Además, nos centraremos en si es factible incorporar el flamenco en los distintos territorios del estado, ya que es considerado «una de las músicas autóctonas más importantes del planeta» (López, 2004, p. 9).

Así, en este texto se interpreta la didáctica del folclore musical español en las distintas comunidades autónomas como el acercamiento a esta parte del patrimonio musical en la escuela, promoviendo la sensibilización de los niños y niñas. Del mismo modo, esta didáctica se divide en cada comunidad autónoma en una específica, como en Aragón sería la didáctica de la jota aragonesa o en Andalucía la didáctica del flamenco, entendida como "una aproximación al mismo, partiendo de sus características musicales y literarias [...] realizada desde una posición científica para su aplicación en el ámbito escolar" (Cenizo, 2009, p. 23).

\section{El folclore en los currículos de segundo ciclo de Educación Infantil}

En este apartado vamos a intentar resolver dos de las principales preguntas que nos surgen al abordar esta temática: ¿Está capacitado nuestro 
sistema educativo para apostar por la introducción del folclore musical español en las aulas de Educación Infantil? y ¿permiten las diferentes normas autonómicas la introducción de folclore distinto al de la propia comunidad?

\subsection{Andalucía}

La ORDEN de 5 de agosto de 2008, por la que se desarrolla el Currículo correspondiente a la Educación Infantil en Andalucía incluye en uno de sus principios el trabajo de manera transversal de:

El patrimonio cultural y natural de nuestra comunidad, su historia, sus paisajes, su folklore, las distintas variedades de la modalidad lingüística andaluza, la diversidad de sus manifestaciones artísticas: música, literatura, pintura..., tanto tradicionales como actuales, así como las contribuciones de sus mujeres y hombres a la construcción del acervo cultural andaluz (BOJA n. ${ }^{\circ}$ 169, 2008, p. 18).

Además, el currículo andaluz de Educación Infantil hace especial hincapié en el flamenco. Esto es así puesto que, de acuerdo con López (2004), «es necesario y coherente llevarlo a las escuelas, porque supone encontrarse con las raíces de un pueblo y una cultura. El flamenco es, tal vez, la manifestación cultural más representativa de la idiosincrasia de lo andaluz» (p. 9). De hecho, es una realidad/su introducción en las aulas de Educación Infantil de Andalucía.

Sin embargo, pese a que este currículo expone que «los contenidos relacionados con el medio natural y el acervo cultural de Andalucía deberán contemplarse en el currículo para que sea conocido, valorado y respetado como patrimonio propio y en el marco de la cultura española y universal» (BOJA n. ${ }^{\circ}$ 169, 2008, p. 23), también podemos intuir la incorporación de otros folclores musicales españoles si consideramos que la sociedad a la que pertenece el alumnado no es solo la andaluza sino también la española, ya que «el conocimiento del patrimonio cultural de la sociedad a la que pertenecen contribuye en los niños y niñas a la construcción de su identidad personal 
aumentando y definiendo su sentimiento de pertenencia a una sociedad y cultura determinada» (BOJA n. ${ }^{\circ}$ 169, 2OO8, p. 30), como se expone en el Área 2 de "Conocimiento del entorno".

\subsection{Aragón}

En la Comunidad Autónoma de Aragón encontramos la ORDEN de 28 de marzo de 2008, del Departamento de Educación, Cultura y Deporte, por la que se aprueba el currículo de la Educación Infantil y se autoriza su aplicación en los centros docentes de la Comunidad Autónoma de Aragón, donde uno de los objetivos del Área II de "Conocimiento del entorno" hace referencia a "conocer las fiestas, tradiciones, folclore y costumbres de Aragón y participar en las de su entorno, disfrutar con ellas y valorarlas, estando abiertos a otras manifestaciones culturales» (BOA n. ${ }^{\circ}$ 43, 2008, p. 4964), además de favorecer el conocimiento, el interés, la valoración y el disfrute con el folclore de Aragón.

En relación con la introducción de folclore musical de otras comunidades autónomas, el currículo aragonés de Educación Infantil muestra con contundencia que es un hecho posible:

En estos primeros años será fundamental que la intervención educativa se dirija a conseguir que el alumnado sienta la música y desarrolle el placer por escucharla y producirla, potenciando a la vez su valor como instrumento de apropiación cultural de tradiciones y formas de expresión propias de la Comunidad autónoma de Aragón, así como de otras relacionadas con otros lugares (BOA n. $.^{\circ} 43,2008$, p. 4970).

\subsection{Canarias}

En Canarias, el DECRETO 183/2008, de 29 de julio, por el que se establece la ordenación y el currículo del $2^{\circ}$ ciclo de la Educación Infantil en la Comunidad Autónoma de Canarias, manifiesta en el Área "Conocimiento del entorno" que «el conocimiento y valoración del entorno inmediato podría favorecer el interés por conocer y valorar otros entornos menos cercanos que también han de ser objeto de trabajo en la escuela y fuera de ella» (BOC n. ${ }^{\circ}$ 163, 


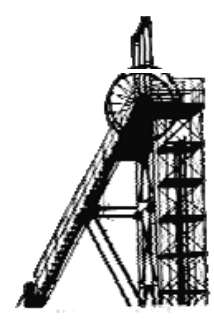

p. 15993). Así, tanto el folclore musical canario, como el de otras comunidades autónomas puede ser introducido en las aulas insulares. Además, en el Área “Lenguajes: comunicación y representación" de esta norma se estipula que:

[...] adquiere especial relevancia la utilización de la música tradicional, con la que se pueden desarrollar capacidades perceptivas, de expresión vocal, instrumental y de movimiento, así como actitudes de valoración y respeto por el propio patrimonio cultural y el de otras regiones o países presentes en su entorno (BOC n. ${ }^{\circ} 163$, p. 16000).

\subsection{Cantabria}

En el Decreto 79/2008, de 14 de agosto por el que se establece el currículo del segundo ciclo de Educación Infantil en la Comunidad Autónoma de Cantabria, se hace referencia a la necesidad de incorporar en el currículo de dicho ciclo experiencias encaminadas a «promover el conocimiento y la valoración, por parte del alumnado, del patrimonio natural y cultural del entorno más próximo» (BOC n. ${ }^{\circ}$ 164, 2008, p. 11544). Es decir, se trabajarán en el aula elementos del patrimonio cultural propios de la sociedad cántabra, como entorno próximo, incluyéndose elementos de dicho folclore en forma de danzas o canciones, entre otras representaciones.

Asimismo, en este decreto se afirma que «en este ciclo, en el mismo contexto de acercamiento al mundo se produce el conocimiento, comprensión, uso y valoración de las diferentes manifestaciones culturales y artísticas de distintas tradiciones, contribuyendo al desarrollo de la competencial cultural y artística» (BOC n. $.164,2008$, p. 11547), lo que hace posible el tratamiento del folclore musical/de otras comunidades autónomas como parte del patrimonio cultural que los alumnos de esta etapa pueden conocer. Este tratamiento se enfocará siempre «desde una perspectiva abierta e integradora que les permita conocer diversos modos y manifestaciones culturales presentes en la sociedad» (BOC n. ${ }^{\circ} 164,2008$, p. 11550). 


\subsection{Castilla y León}

En la Comunidad Autónoma de Castilla y León, la normativa educativa vigente es el DECRETO 122/2007, de 27 de diciembre, por el que se establece el currículo del segundo ciclo de la Educación Infantil en la Comunidad Autónoma de Castilla y León, que recoge entre los contenidos del Área III de "Lenguajes: comunicación y representación" uno relacionado con el fomento en el alumnado la «curiosidad por las canciones y danzas de nuestra tradición cultural y de otras culturas» (BOCyL n. ${ }^{\circ} 1,2008$, p. 16). El hecho de tratar este contenido en las aulas del segundo ciclo nos lleva a confirmar dos ideas: por un lado, que desde el currículo se favorece el conocimiento del folclore musical castellanoleonés; y, por otro lado, que es posible la introducción de folclore musical de otras comunidades autónomas en estas aulas de Educación Infantil, ya que habla de generar curiosidad por canciones y danzas de otras culturas, como puede ser la aragonesa o la navarra, entre todas las demás.

\subsection{Castilla-La Mancha}

En el Decreto 67/2007, de 29-05-2007, por el que se establece y ordena el currículo del segundo ciclo de la Educación Infantil en la Comunidad Autónoma de Castilla-La Macha encontramos referencias al «conocimiento, valoración y la conservación del patrimonio histórico, artístico, cultural y natural de la Comunidad Autónoma de Castilla-La Mancha» (DOCM n. ${ }^{\circ}$ 116, 2007, p. 14744), incluyéndose así el folclore musical propio de esta comunidad.

Del mismo modo, el tratamiento del folclore de otras comunidades autónomas es posible debido a un contenido del Área de "Conocimiento e interacción con el entorno" que estipula la «valoración y respeto por otras lenguas y cultura generando actitudes de confianza y aprecio» (DOCM n. ${ }^{\circ} 116$, 2007, p. 14751). 


\subsection{Cataluña}

En Cataluña, el DECRETO 181/2008, de 9 de septiembre, por el que se establece la ordenación de las enseñanzas del segundo ciclo de la educación infantil no hace referencias explícitas al folclore musical catalán, como la sardana, pero sí contempla su didáctica debido a la «identificación de fiestas, tradiciones, historias o leyendas del entorno próximo y de Cataluña» (DOGC n. ${ }^{\circ}$ 5216, 2008, p. 68268) en el Área de "Conocimiento del entorno", ya que el folclore musical no deja de ser parte de la tradición de la comunidad.

Además, esta normativa permite la introducción de folclore de otras comunidades autónomas, puesto que el sexto objetivo para el desarrollo de las capacidades en el segundo ciclo de la Educación Infantil establece «conocer experiencias, historias y símbolos de la cultura propia del país y de la de otros compañeros y compañeras, generando actitudes de confianza y respeto por las diferencias y valorando las relaciones sociales y afectivas que se establezcan» (DOGC n. ${ }^{\circ}$ 5216, 2008, p. 68266).

\subsection{Ciudades Autónomas: Ceuta y Melilla}

La Orden ECI/3960/2007, de 19 de diciembre, por la que se establece el currículo y se regula la ordenación de la educación infantil es la normativa vigente en las Ciudades Autónomas de Ceuta y Melilla. En ella encontramos solo una referencia al folclore, en relación con el conocimiento e interés que se muestre por las manifestaciones culturales del entorno, donde se incluiría el folclore musical de estas ciudades autónomas, así como con el agrado hacia las obras musicales.

Del mismo modo, el folclore musical de otros territorios españoles tiene cabida debido a la «audición activa y reconocimiento de algunas obras musicales de diferentes géneros y estilos. Participación activa y disfrute en la interpretación de canciones, juegos musicales y danzas» (BOE n. ${ }^{\circ}$ 2008, p. 24), contenido del Área 3 de "Lenguajes: comunicación y representación". 


\subsection{Comunidad de Madrid}

En el caso de la Comunidad de Madrid, el DECRETO 17/2008, de 6 de marzo, del Consejo de Gobierno, por el que se desarrollan para la Comunidad de Madrid las enseñanzas de la Educación Infantil establece, dentro de los objetivos del Área III de "Lenguajes: Comunicación y representación”, uno relacionado con «conocer algunas manifestaciones culturales de su entorno» (BOCM n. ${ }^{\circ}$ 61, 2008, p. 14), entre las que se encuentran las relacionadas con el folclore musical propio de la zona, ya que el concepto 'manifestaciones culturales' engloba todos los ámbitos de la cultura, incluidas la música y las danzas. Asimismo, este Área II cuenta con un bloque de contenidos destinado al "Lenguaje musical", donde se señala la «audición atenta de obras musicales presentes en el entorno: canciones populares infantiles, danzas, bailes y audiciones» (BOCM n. ${ }^{\circ}$ 61, 2008, p. 15), así como con «la canción como elemento expresivo. Canciones de entorno y del mundo» (BOCM n. ${ }^{\circ}$ 61, 2008, p. 15), lo que corrobora nuestra idea de que en las aulas madrileñas del segundo ciclo se trabaja el folclore musical de la zona.

Por otra parte, cabe decir que también es viable el tratamiento del folclore musical de otras comunidades autónomas, dado que en el Área III de "Lenguajes: Comunicación y representación", se hace referencia a objetivos como «cantar, escuchar, bailar e interpretar» (BOCM n. ${ }^{\circ}$ 61, 2008, p. 14), y «aprender canciones, bailes y danzas» (BOCM n. ${ }^{\circ}$ 61, 2008, p. 14). Son objetivos generales en los que puede tener cabida el folclore musical de otras partes del territorio español, ya que no se especifica que estas canciones, bailes y danzas deban ser del entorno próximo. Asimismo, en el bloque de contenido relativo al “Lenguaje musical" se hace referencia a «la canción como elemento expresivo. Canciones de su entorno y del mundo» (BOCM n. ${ }^{\circ}$ 61, 2008, p. 15), donde se hace evidente el tratamiento del folclore musical de otras parte del mundo y, en este caso, también el de otras comunidades autónomas. 


\subsection{Comunidad Foral de Navarra}

El DECRETO FORAL 23/2007, de 19 de marzo, por el que se establece el currículo de las enseñanzas del segundo ciclo de la Educación Infantil en la Comunidad Foral de Navarra, establece en el tercer bloque del Área de “Lenguajes: comunicación y representación", un contenido relacionado con la «audición activa de obras musicales presentes en el entorno. Participación activa y disfrute en la interpretación de canciones, juegos musicales y danzas» (BON n. ${ }^{\circ}$ 51, 2007, p. 4513), por lo que podemos afirmar que el folclore navarro sí que se trabaja en las aulas de este segundo ciclo de Educación Infantil.

Ahora bien, esta misma referencia curricular nos sirve para determinar que también es posible la introducción en dichas aulas del folclore musical de otras comunidades autónomas, puesto que las obras musicales presentes en el entorno a las que hace referencia el contenido, bien pueden interpretarse como propias del territorio navarro o de cualquier parte de España.

\subsection{Comunitat Valenciana}

En la Comunitat Valenciana, el DECRETO 38/2008, de 28 de marzo, del Consell, por el que se establece el currículo del segundo ciclo de la Educación Infantil en la Comunitat Valenciana, establece como uno de los objetivos de ciclo «conocer y apreciar las manifestaciones culturales de su entorno, mostrando interés y respeto hacia ellas, así como descubrir y respetar otras culturas próximas» (DOCV n. . 5734, 2008, p. 55019). Asimismo, en el Área III de "Lenguajes: comunicación y representación" hay un bloque dedicado a la "Expresión musical" en el que se señalan contenidos como «el conocimiento de las canciones populares infantiles, danzas, canciones del folklore, canciones contemporáneas, baile y audiciones» (DOCV n. ${ }^{\circ}$ 5734, 2008, p. 55047), lo que significa que en las aulas de este ciclo se presta atención al folclore propio, permitiendo al alumnado conocer la cultura musical valenciana. 
En relación con la posibilidad de introducir elementos del folclore musical de otras comunidades autónomas, cabe decir que es posible gracias a la presencia en el currículo de objetivos como el mencionado anteriormente, en el que se hace referencia al descubrimiento y respeto de elementos de otras culturas próximas, como son los de otras zonas del territorio español.

\subsection{Extremadura}

El DECRETO 4/2008, de 11 de enero, por el que se aprueba el Currículo de Educación Infantil para la Comunidad Autónoma de Extremadura recoge como uno de los objetivos generales «iniciarse en la participación y descubrimiento de las manifestaciones culturales propias de la Comunidad Autónoma» (DOE n. ${ }^{\circ}$ 12, 2008, p. 1230), entre las que se encuentran elementos del folclore musical de la zona. Esto se concreta todavía más al establecer, para el segundo ciclo de la etapa, que los alumnos deben desarrollar capacidades que les permitan «conocer las manifestaciones culturales propias de la Comunidad Extremeña» (DOE n. ${ }^{0}$ 12, 2008, p. 1231), lo que implica, como se recoge en el Área de "Conocimiento del entorno" la «valoración y respeto del patrimonio artístico y cultural de nuestra Comunidad Autónoma» (DOE n. ${ }^{0}$ 12, 2008, p. 1264). Asimismo, en el Área de "Los lenguajes: comunicación y representación" se destina un bloque al "Lenguaje musical2 y se establecen contenidos relacionados con la «valoración e interés por el folklore extremeño» (DOE n. ${ }^{\circ}$ 12, 2008, p. 1271) y las «canciones del folklore extremeño, canciones contemporáneas, danzas populares, bailes regionales» (DOE n. ${ }^{\circ} 12,2008$, p. 1271). Así, este último contenido permite el tratamiento del folclore de otras comunidades autónomas, englobado dentro de danzas populares y bailes regionales, sin olvidarnos del «disfrute del canto, la danza, el baile y la interpretación musical» (DOE n. ${ }^{\circ}$ 12, 2008, p. 1271), que no determina que sea del entorno cercano o de otras zonas del territorio español. 


\subsection{Galicia}

En el caso de Galicia, el Decreto 330/2009, de 4 de junio, por el que se establece el currículo de la educación infantil en la Comunidad Autónoma de Galicia, establece en su Área de "Conocimiento del entorno" un objetivo relacionado con «apreciar y hacer suyos algunos elementos significativos propios de la tradición y de la cultura gallega - expresiones artísticas, costumbres, fiestas populares, folclore, gastronomía, etc.» (DOG n. ${ }^{\circ} 121,2009$, p. 10789), lo que nos lleva a pensar que el folclore musical gallego sí que tiene cabida en las aulas del segundo ciclo de esta etapa.

Acerca de la didáctica del folclore de otras comunidades autónomas, cabe decir que en el currículo gallego no se hace referencia como tal a este hecho, si bien aparecen contenidos del Área de "Lenguajes: comunicación y representación" relativos a la «audición atenta de obras musicales populares, clásicas y contemporáneas. Participación activa y disfrute en la interpretación de canciones, juegos musicales y danzas colectivas e individuales» (DOG n. ${ }^{\circ} 121,2009$, p. 10794). Estas músicas populares a las que se refiere bien pueden ser las propias de otras comunidades autónomas, dada su relevancia en el resto de España.

\subsection{Islas Baleares}

Pese a que en su contenido no menciona explícitamente ni el folclore ni el patrimonio, el Decreto 71/2008, de 27 de junio, por el cual se establece el currículo de la educación infantil en las Islas Baleares sí que permite el tratamiento didáctico del folclore musical propio de la comunidad y de otras partes del estado puesto que uno de sus objetivos es «disfrutar de las manifestaciones culturales presentes en el entorno, conocer las más identificativas de las Islas Baleares y respetar las de otros lugares» (BOIB n. ${ }^{o}$ 92, 2008, p. 179). Además, incide en que «el profesorado, para contribuir al mantenimiento de la identidad propia de las Islas Baleares, tiene que prever el 
tratamiento de la cultura y las tradiciones en las diferentes áreas» (BOIB n. ${ }^{\circ}$ 92, 2008, p. 179).

Es interesante, también, una reflexión presente en este currículo que consideramos digna de destacar:

Ser enseñante de educación infantil, hoy, precisa de un perfil de alta complejidad y de gran responsabilidad. Requiere tener competencias culturales, pedagógicas, psicológicas, metodológicas y didácticas, además de una sensibilidad abierta y la disposición personal madura y responsable hacia las relaciones educativas con los niños y las familias. Supone formarse permanentemente para mejorar la escuela y la propia práctica, para abordar los problemas específicos de la actividad y para el crecimiento personal (BOIB n. ${ }^{\circ}$ 92, 2008, p. 182).

\subsection{La Rioja}

El DECRETO 25/2007, de 4 de mayo, por el que se establece el Currículo del Segundo Ciclo de Educación Infantil en la Comunidad Autónoma de La Rioja tampoco nombra el folclore o el patrimonio, ni cercano ni ajeno. Sin embargo, sí que es posible una didáctica del folclore musical propio, la jota riojana, por ejemplo, como de otras comunidades autónomas. Esto es debido a que uno de los objetivos que dicta esta normativa es «conocer y apreciar las manifestaciones culturales y artísticas propias de nuestra Comunidad Autónoma y del territorio nacional, mostrando interés y respeto hacia ellas» (BOR n. ${ }^{\circ}$ 62, 2007, p. 3288), la cual también expone que «el alumnado utilizará como recurso didáctico las tradiciones culturales de La Rioja y valorará también las tradiciones españolas como parte de la riqueza cultural común» (BOR n. ${ }^{\circ}$ 62, 2007, p. 3290).

\subsection{País Vasco}

En el País Vasco, el DECRETO 237/2015, de 22 de diciembre, por el que se establece el currículo de Educación Infantil y se implanta en la Comunidad Autónoma del País Vasco incorpora que en el segundo ciclo de esta etapa se 


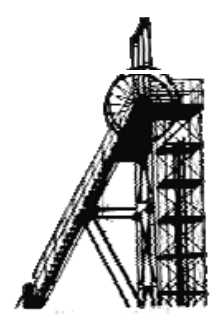

deben realizar actividades de tradición cultural dentro de las familias de situaciones relacionadas con el Ámbito del Conocimiento de la propia identidad y del medio físico y social.

Dentro del “Ámbito de la construcción de la propia identidad y de la comunicación y representación" aparecen dos contenidos que nos permiten tratar didácticamente el folclore musical vasco, como el zorcico, y el de otras comunidades autónomas: «audición de algunas obras musicales de diferentes géneros y estilos» (BOPV n. ${ }^{\circ}$ 9, 2016, p. 45) y «valoración e interés por el folklore y otras manifestaciones artísticas de su entorno cultural» (BOPV n. ${ }^{\circ}$ 9, 2016, p. 45). Además, también se refleja en el criterio e indicador de evaluación: «muestra atención e interés ante manifestaciones culturales diversas y hacia las producciones de las demás personas» (BOPV n. ${ }^{\circ}$ 9, 2016, p. 47).

\subsection{Principado de Asturias}

En el caso del Principado de Asturias, el DECRETO 85/2008, de 3 de septiembre, por el que se establece el currículo del segundo ciclo de Educación Infantil fija como uno de sus objetivos de etapa «descubrir y participar en algunas manifestaciones sociales, culturales y artísticas de su entorno desarrollando una actitud de interés y aprecio hacia el patrimonio cultural asturiano» (BOPA n. ${ }^{\circ}$ 212, 2008, p. 20442). Dentro de este patrimonio se encuentran incluidas las manifestaciones del folclore asturiano, como el «reconocimiento de señas de identidad cultural asturiana (himno de Asturias, canciones, la bandera, las fiestas, las tradiciones, los mitos, etcétera) e interés por participar en actividades sociales y culturales» (BOPA n. '212, 2008, p. 20450).

Por su parte, sí que se puede hablar del tratamiento del folclore de otras comunidades autónomas, ya que en el Área de "Lenguajes: comunicación y representación" se determinan contenidos acerca de la «audición atenta de obras musicales. Participación activa y disfrute en la interpretación de canciones, juegos musicales y danzas, prestando especial atención a las 
pertenecientes a nuestra tradición cultural» (BOPA n. ${ }^{\circ} 212,2008$, p. 20454). Esto es así dado que consideramos que la tradición cultural de la que se habla puede ser la española, englobando así cualquier tipo de folclore musical del país.

\subsection{Región de Murcia}

En la Región de Murcia se puede observar claramente la didáctica del folclore musical murciano, como las parrandas murcianas, en el Decreto número 254/2008, de 1 de agosto, por el que se establece el currículo del Segundo Ciclo de la Educación Infantil en la Comunidad Autónoma de la Región de Murcia, en el contenido del Área "Conocimiento del entorno" relacionado con «costumbres, folklore y otras manifestaciones culturales y artísticas de la Región de Murcia» (BORM n. ${ }^{0}$ 182, 2008, p. 24969), entre otros apartados curriculares.

Además, esta norma también hace posible la introducción de folclore de otras comunidades autónomas debido a otro contenido del Área "Lenguajes: Comunicación y representación" que expone la «curiosidad por las canciones y

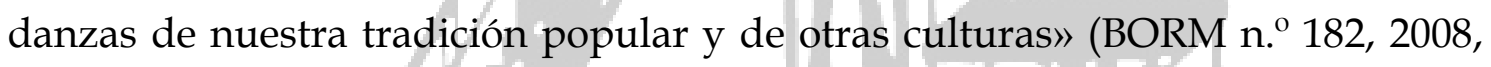
p. 24972). En este caso, se puede justificar ya que la tradición popular a la que se refiere el currículo puede ser la española, pero si consideramos que es la murciana, el resto de culturas españolas también se encuentran implícitas en este contenido.

\section{El caso del flamenco}

Después de este análisis en relación al folclore musical en los distintos currículos del segundo ciclo de Educación Infantil presentes a lo largo y ancho de la geografía española, vamos a centrarnos en uno de los folclores españoles más conocidos y apreciados dentro y fuera de nuestras fronteras: el flamenco.

De acuerdo con López (2009), «en la medida en que la escuela debe ser un lugar de encuentro con la cultura, un lugar de reflexión sobre el conocimiento 


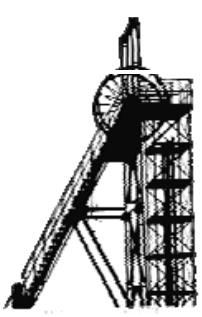

heredado, es necesario que el flamenco llegue a ella» (pp. 9-10). Por ello, la didáctica del flamenco es un fenómeno que actualmente se practica en el sistema educativo andaluz, desde la etapa de Educación Infantil hasta la Universidad.

Sin embargo, esta didáctica se podría llevar a cabo en las aulas del resto de territorios del país, entre otras cosas, por su condición de folclore. Sí, como hemos admitido al principio de este artículo, muchos de los lectores que han llegado hasta este punto no consideran folclore al flamenco, entre otras cosas porque:

Aunque el Flamenco bebe de las fuentes del folclore [...], se distingue de éste por su mayor elaboración musical y por el carácter individual de la interpretación, que lo elevan a la categoría de arte. Los estilos flamencos han nacido, evolucionando, de formas folclóricas, y no sólo los llamados aflamencados, como la petenera, la rondeña, la bambera, campanilleros, el garrotín, etc., sino incluso los más jondos o puros como la toná o la soleá (Cenizo, 2009, p. 19).

Pese a esta diferencia con algunos lectores, concibamos o no el flamenco como folclore, estaremos todos de acuerdo en que sí que podemos situarlo dentro de la cultura española y como parte del patrimonio cultural y musical del país. De hecho, ambas concepciones permiten su incorporación en todas las aulas españolas del segundo ciclo de Educación Infantil.

Es más, la declaración del flamenco como Patrimonio Cultural de la Humanidad por parte de la UNESCO abre otra vía para su introducción en todo el sistema educativo español, sobretodo, y a nuestro modo de ver, en las etapas de Educación Primaria y Educación Secundaria Obligatoria.

\section{Didáctica del flamenco en Educación Infantil}

Llegados a este punto, es necesario tratar también un tema trascendental que nos ocupa: la didáctica del flamenco en Educación Infantil. No debemos olvidar que merece gran importancia saber cómo llevar a cabo la introducción del flamenco, con qué recursos y con qué actividades. Pero para lograr esta 
didáctica específica tampoco se puede descuidar la formación de base de los maestros (tanto en la universidad como en la formación a lo largo del ejercicio docente), puesto que es, quizá, el primer paso para realizarlo conscientemente y no dejarlo al azar.

Realmente consideramos que existe una didáctica del flamenco para cada etapa educativa, las cuales poseerán elementos comunes pero también diferenciadores. En nuestro caso entendemos la didáctica del flamenco propia de Educación Infantil como el acercamiento pedagógico al mismo en las aulas de esta etapa educativa. De acuerdo con Cenizo (2009), esta es la etapa propicia para su introducción debido a que:

Los niños aún están más cerca de lo popular a través de las canciones infantiles, por esto quizá resulte más fácil acercarlos a la melodía flamenca siguiendo las directrices que han indicado algunos profesores y flamencólogos, como Manuel Herrera Rodas, Ricardo Rodríguez Cosano o Caty León Benítez, y otros que se le han sumado con los años, como Jerónimo Utrilla (pp. 23-24).

En consecuencia, los objetivos de la didáctica del flamenco en Educación Infantil podrían ser, entre otros:

- Introducir progresivamente contenidos de flamenco en las aulas de esta etapa educativa.

- Dar a conocer al alumnado diferentes palos flamencos tales como las bulerías, los fandangos, los tangos, etc.

- Promover el interés por el conocimiento del folclore andaluz.

- Facilitar la identificación de diferentes instrumentos propios del flamenco como las castañuelas, el cajón o la guitarra.

- Acercar al alumnado diferentes elementos característicos de la indumentaria andaluza tales como los trajes, abanicos o mantones. 


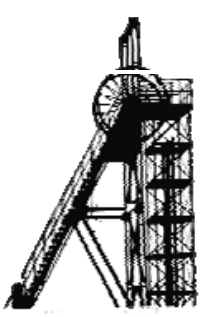

- Fomentar progresivamente el interés del alumnado por la escucha activa y atenta de piezas flamencas.

Pero además, en el momento de reflexión previo a la introducción de este folclore en el aula de Educación Infantil, el maestro debe planificar conscientemente qué se pretende enseñar y transmitir. De hecho, «del flamenco, como de cualquier manifestación cultural, se puede aprender todo, pasar de puntillas por él, o simplemente pasar y dejar que sean otros los que después vengan a enseñarlo» (Valero, 2004, p. 79).

Después de fijar qué se desea enseñar, es conveniente estudiar cómo se desea hacer. Para ello podemos seguir la clasificación desarrollada por Rueda (2004), en la que tras las actividades de motivación y de efecto (experiencias flamencas) tendrían cabida las actividades adicionales, tales como las que implican movimiento corporal o elementos flamencos, auditivas, con instrumentos, de visionado de vídeos o de representación. Además, tampoco podemos olvidar la evaluación, tanto del alumnado como de la planificación y el desarrollo de nuestro propio trabajo docente.

Sin embargo, los cimientos de esta didáctica del flamenco en Educación Infantil comienzan con el interés y gusto de los maestros hacia el mismo y la música tradicional andaluza. Esto, junto con el aprendizaje en la Universidad o en otros momentos dentro de la continua formación que debemos realizar los maestros, propician un caldo de cultivo óptimo para su introducción en las aulas de Educación Infantil.

De acuerdo con Valero (2004), la Educación Infantil es «un periodo esencialmente de acercamiento y sensibilización» (p. 80) hacia el flamenco. Este hecho hay que tenerlo siempre en cuenta puesto que la intención principal no es la de crear cantaores, bailaores o tocaores, sino introducirlos en un folclore que ha traspasado fronteras y que supone un ejemplo a seguir para otros folclores españoles. 
Además, la didáctica del flamenco se ha vuelto internacional, puesto que existen numerosos profesores de enseñanza de Español como Lengua Extranjera que la usan en sus clases, quedando expuesto en diferentes congresos y artículos científicos.

Finalmente, no podemos olvidar que la práctica de flamenco:

posee una gran importancia en el desarrollo integral del alumnado en edades tempranas. Cuando se hace una práctica continuada de esta disciplina, el alumnado desarrolla de forma óptima capacidades físicas, mentales y emocionales. [...] Es por tanto, que la danza, siendo un elemento educativo esencial para el desarrollo global del alumnado, sea valorada y conocida como un recurso con gran potencial educativo (Padial-Ruz et al., 2019, p. 397).

\section{Sugerencias para un tratamiento didáctico del flamenco}

A continuación proponemos una serie de reflexiones para el tratamiento didáctico del flamenco en las aulas españolas de Educación Infantil, siempre desde el prisma y el conocimiento del currículo aragonés propio de nuestra comunidad.

De esta manera, debido a su condición de Patrimonio Cultural de la Humanidad podemos afirmar que la introducción del flamenco en el aula es un derecho del alumnado, de tal manera que puedan conocer el folclore, la cultura y la identidad de otras partes del territorio español y, en este caso concreto, de Andalucía. Sin embargo, tras la revisión de los diferentes currículos, podemos afirmar que en ningún momento se hace referencia a elementos del Patrimonio Cultural de la Humanidad ni a la forma de trabajarlos en el aula.

En cualquier caso, este tratamiento del flamenco en el aula implica una serie de obligaciones, entre las que se encuentra la formación permanente y continua del profesorado en materia de Patrimonio Cultural, de modo que, como señala el Plan Nacional de Educación y Patrimonio (PNEyP), se potencie la competencia profesional del docente en este ámbito (MECD, 2015). 


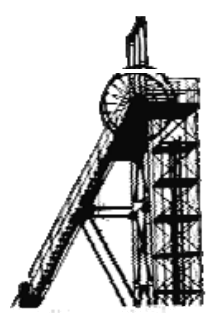

En relación con el PNEyP, este derecho del alumnado a recibir formación acerca del flamenco cobra todavía más relevancia debido a

la necesidad de concienciar desde las primeras fases educativas, de modo que el aprendizaje del alumnado forme parte de un crecimiento integral, físico e intelectual, en el que la convivencia con el Patrimonio, su valoración y conservación sea algo integrado en la vida cotidiana (MECD, 2015, p. 32).

Como consecuencia, hacemos nuestras las palabras de Valero: «basta, pues, de argumentos que nos sirvan de excusa. Encaremos la cuestión de frente, y dispongámonos a la aventura de aprender y de enseñar-siempre apasionante- el arte flamenco, que, además, nos resultará divertido» (2004, p.80).

Por ello, y como forma de potenciar dicha diversión, planteamos una serie de actividades con las que dar a conocer las posibilidades del flamenco en las aulas de Educación Infantil, siempre desde la perspectiva de las tres áreas de aprendizaje que incluyen los correspondientes currículos nacionales. Dada la necesidad de que el aprendizaje sea globalizado y los contenidos estén interrelacionados, las siguientes propuestas implican un enfoque integral para el desarrollo de las tres áreas en cuestión:

- Elaborar un mural sobre flamenco en el que se recabe información acerca de los instrumentos que se utilizan y en qué familias se clasifican (cuerda pulsada, percusión...), algunos ejemplos de palos flamencos, las características del baile, así como fotografías de la indumentaria tradicional o de grupos flamencos que existan.

- Investigar acerca de personas relevantes en el mundo del flamenco, prestando especial atención a las figuras femeninas, para después incluir la información obtenida en un libro de aula sobre el flamenco, el cual quedará disponible en la biblioteca para que los alumnos hagan uso de él cuando sea necesario. 
- Dedicar un día en el aula a conocer la indumentaria tradicional, de modo que en un primer momento se busque información sobre dichos trajes en el ordenador, en libros y otros soportes para, después, crearlos a partir de materiales reciclados (bolsas de basura, ropas traídas de casa, disfraces que haya en el aula en la zona de juego simbólico...).

- Destinar un espacio del aula al flamenco, creando así un "rincón del flamenco" en el que se vayan organizando todos los materiales elaborados en clase y recopilando las informaciones obtenidas.

- Leer coplas flamencas sencillas en el aula y analizarlas, de modo que entiendan el significado de cada una de ellas y que puedan llegar a memorizarlas y recitarlas tanto a los compañeros de otros cursos como a las familias.

- Escuchar audiciones de diferentes palos flamencos para discriminar entre música y ruido, marcar el pulso y el acento, identificar instrumentos musicales (guitarra, cajón, castañuelas...), improvisar sobre una base flamenca, interpretar canto y baile flamenco, etc.

- Dramatización de diferentes palos flamencos haciendo uso de expresiones faciales y del cuerpo, de modo que se desarrolle la motricidad tanto fina como gruesa, la lateralidad, la improvisación, etc.

- Realizar adaptaciones de cuentos sobre flamenco ya existentes, como por ejemplo "Juanito el jabegote" o "Salvador Rueda: El niño que no sabía escribir sus poemas" ambos de Miguel López Castro, para que se ajusten al nivel de comprensión lectora del alumnado de Educación Infantil y puedan ser utilizados en dichas aulas. 


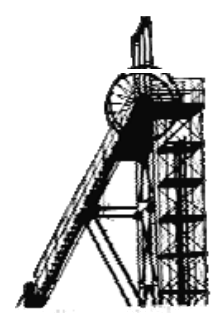

- Crear nuevas historias y cuentos cuyo tema central sea el flamenco, especialmente si tenemos en cuenta la escasez de recursos de este tipo que hay disponibles.

- Recibir visitas en el aula para que personas que tengan conocimientos sobre flamenco, desciendan de Andalucía o hayan estudiado sobre este folclore transmitan sus conocimientos al alumnado. Asimismo, estas visitas se pueden complementar con un tiempo de preguntas y respuestas en el que los alumnos expresen sus curiosidades y pregunten sobre aquello que más llama su atención.

Estas aportaciones también deben ir orientadas al ámbito familiar, favoreciendo así el principio de coherencia educativa por el cual familia y escuela trabajan de manera coordinada y cohesionada con el fin de favorecer el proceso de enseñanza-aprendizaje. Esto es así dado que en el aprendizaje y desarrollo del alumnado influyen ambos contextos, familia y escuela, especialmente si tenemos en cuenta que es con el primero de ellos con quien interactúan y realizan aprendizajes desde el momento del nacimiento. En este sentido, consideramos que una forma de implicar a las familias en el aprendizaje podría ser por medio de una "semana de la familia flamenca", de modo que en casa realicen una serie de actividades que los maestros determinen y conozcan esta cultura y folclore con sus hijos, respaldando el trabajo que se lleva a cabo en el aula. Es decir, se trataría de que las familias conozcan también la cultura y el folclore andaluz por medio de retos que deben ir realizando durante toda la semana, siendo algunos ejemplos: buscar información sobre el flamenco, sus cantes, bailes e instrumentos más característicos y, por ejemplo, que dibujen y pinten los diferentes instrumentos; ver vídeos de cante y baile flamenco para compararlo con el folclore de la propia comunidad, siendo así más conscientes de las similitudes y diferencias; o 
practicar una copla hasta memorizarla para después compartirla con el resto de compañeros en clase.

Una vez expuestas nuestras aportaciones, nos gustaría recordar las palabras de Valero (2004):

El aprendizaje del flamenco es una labor a largo plazo, por lo que no cabe exigir unos resultados inmediatos, sino que nos hemos de proponer como objetivo primero y esencial que alumnas y alumnos se enamoren de él, que queden, en definitiva, "atrapados" en su música y en su poesía (p. 80).

Consideramos que el aprendizaje de (y a través del) flamenco debe iniciarse desde edades tempranas, como venimos defendiendo a lo largo de todo el texto, pero también debe tener continuidad en el resto de etapas educativas, ya que solo así se asegura un aprendizaje significativo, la profundización en los contenidos tratados y la especialización del alumnado, en la medida de lo posible, en este folclore. Ahora bien, en la etapa que nos ocupa, que es la de Educación Infantil, el tratamiento didáctico del flamenco tiene como principal objetivo el acercamiento y la sensibilización del alumnado, que es lo que pretendemos conseguir con las propuestas de trabajo planteadas.

\section{Inconvenientes para un tratamiento didáctico del flamenco}

Si bien es cierto que hasta ahora hemos hablado de los beneficios de introducir el flamenco en el aula, así como de posibles propuestas para ello, es momento de centrar nuestra atención en los inconvenientes o dificultades que pueden frenar a los profesionales de la educación de todo el territorio a la hora de introducir estos contenidos en su aula.

En primer lugar, cabe hacer referencia a la gran variedad de materiales literarios que señalan a nivel teórico los elementos del flamenco que se pueden trabajar en el aula, como puede ser el cante, el baile, la guitarra flamenca, etc. Sin embargo, son pocos los materiales didácticos y las propuestas prácticas, ya 


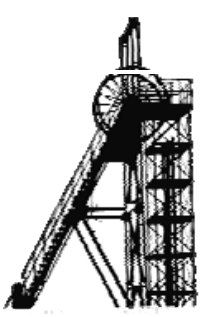

sea en forma de Unidades Didácticas, talleres o proyectos, entre otros, que desarrollen paso a paso actividades con las que trabajar dichos contenidos teóricos. Como consecuencia, docentes de comunidades autónomas diferentes a la andaluza pueden encontrar en este hecho un gran inconveniente, dado que pueden desconocer este folclore y la forma de tratar sus elementos principales en el aula de Educación Infantil. Es por este motivo por lo que consideramos fundamental que todas las actividades, propuestas y materiales didácticos disponibles se organicen y se pongan a disposición de los docentes de todo el territorio español, de manera que puedan acceder fácilmente a ellos y no haya barreras a la hora de introducir el flamenco en el aula. Por el mismo motivo, anteriormente hemos estimado oportuno plantear una serie de ideas y ejemplos de actividades que faciliten la labor del profesorado y permitan un progresivo tratamiento del flamenco en las aulas de esta etapa educativa.

Otro de los inconvenientes, a nuestro parecer, reside en la formación universitaria que reciben los maestros de Educación Infantil, puesto que en una sociedad intercultural como es la nuestra, en el itinerario de dichos estudios universitarios debería tener cabida el análisis de otras culturas diferentes a la de la propia comunidad, así como su tratamiento en las aulas de esta etapa. Es por este motivo por lo que, en el caso que nos ocupa, los maestros pueden tener un gran desconocimiento del flamenco que les impida tratarlo en el aula de manera óptima, prefiriendo no darle cabida ni en el horario lectivo ni en las programaciones didácticas. Sin lugar a dudas, podemos relacionar esta idea con la ya mencionada anteriormente acerca de la formación permanente y continua como obligación del profesorado.

Unido a esta necesidad formativa, cabe decir que el hecho de que no haya profesorado especialista en flamencología en las diferentes comunidades autónomas todavía dificulta más la introducción de dicho folclore en el aula. Como ya hemos mencionado, los maestros de Educación Infantil normalmente carecen de formación en este ámbito de conocimiento, lo que unido al trabajo 
que desarrollan en las aulas sobre el folclore de su comunidad, provoca que no dediquen tiempo a formarse en flamenco y se haga evidente la necesidad de que haya profesores especialistas que orienten su práctica docente.

Además, en este proceso de enseñanza-aprendizaje influye el bagaje musical del alumnado, que evidentemente no suele escuchar audiciones de folclore musical español, como puede ser el flamenco, que nos ocupa en este momento, o la jota, que tanto nos representa a los aragoneses. En cambio, en su día a día prima la audición de canciones más comerciales y géneros musicales muy diferentes a los que estamos mencionando en el texto, como puede ser el pop, el reggaetón o el rock, entre otros. Por este motivo, resulta complicado que los alumnos sean capaces no solo de escuchar, sino de disfrutar de audiciones de folclore tradicional nacional.

\section{Conclusiones}

En primer lugar, es necesario admitir que el reflejo en estas páginas del análisis realizado no ha sido pormenorizado por no molestar al lector con un denso estudio plagado de citas que pudiera llevar a una situación de hastío. Por esta razón, animamos a quien esté interesado en esta temática a realizar la lectura y análisis del currículo de la comunidad autónoma que le concierna para justificar la introducción de la didáctica del folclore en su aula de Educación Infantil.

Después de este análisis efectuado, consideramos que nuestro sistema educativo, indiferentemente de la comunidad autónoma, está más que capacitado para apostar por una didáctica del folclore musical español en las aulas del segundo ciclo de Educación Infantil.

Sin embargo, hemos comprobado que hay diferentes comunidades autónomas que no hacen referencias explícitas al folclore en sus normativas, algo peligroso en nuestra opinión, porque puede favorecer o facilitar el olvido del bagaje cultural propio en un mundo tan globalizado como el actual. Del 


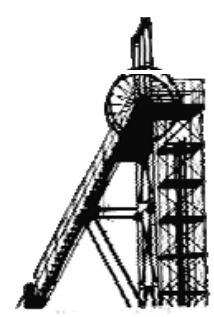

mismo modo, nos sorprende que en algunos currículos no se nombre el patrimonio cultural, tanto autonómico como nacional, o la cultura española en general, especificándose siempre la propia de cada comunidad.

Como maestros defendemos la inclusión del folclore propio de cada comunidad autónoma en el aula de Educación Infantil, pero también creemos posible una sensibilización hacia el resto de folclore musical de nuestro país.

En este sentido, el flamenco, al ser estimado por la población española y por su condición de Patrimonio Cultural de la Humanidad de la UNESCO, parte desde un lugar privilegiado para su trabajo en las aulas de Educación Infantil andaluzas y en las de otras comunidades españolas, como puede ser el caso de la Región de Murcia.

Asimismo, como aragoneses, no podemos olvidarnos de nuestro folclore, $\mathrm{y}$, en especial, de la jota aragonesa, la cual entendemos que debe ser introducida desde la base del sistema educativo aragonés. Eso sí, esta inquietud por la jota se debe promover durante la formación de los nuevos científicos de la educación desde la Universidad. De este modo, abogamos por el desarrollo y promoción de una didáctica de la jota aragonesa en educación formal y no formal tanto dentro como fuera de Aragón.

También sería interesante que el sistema educativo español pusiese en marcha un proyecto de didáctica del folclore interautonómico, creando redes en las que todo el folclore musical interactúe sin importar la ciudad, comarca, provincia o comunidad autónoma en la que se practique.

Finalmente, es importante recalcar que este artículo se centra en la norma, en lo puesto negro sobre blanco, pero como docentes sabemos que la realidad de las aulas dista notablemente de la que dicta el currículo. Por ello la formación, motivación, ganas y valentía de los maestros son indispensables para implementar en un principio una didáctica del folclore autonómico, para, posteriormente, convertirlo en interautonómico. 


\section{Referencias bibliográficas}

CenIzO, José (2009). Poética y didáctica del flamenco: una visión interdisciplinar. Signatura Ediciones de Andalucía, S.L.

DECRETO 122/2007, de 27 de diciembre, por el que establece el currículo del segundo ciclo de la Educación Infantil en la Comunidad de Castilla y León. BOCyL n. ${ }^{\circ}$ 1, $6-16$.

DeCreto 17/2008, de 6 de marzo, del Consejo de Gobierno, por el que se desarrollan para la Comunidad de Madrid las enseñanzas de la Educación Infantil. BOCM n. ${ }^{\circ}$ 61, 6-15.

DECRETO 181/2008, de 9 de septiembre, por el que se establece la ordenación de las enseñanzas del segundo ciclo de la educación infantil. DOGC n. ${ }^{\circ} 5216$, 68256-68272.

DECRETO 183/2008, de 29 de julio, por el que se establece la ordenación y el currículo del $2^{\circ}$ ciclo de la Educación Infantil en la Comunidad Autónoma de Canarias. BOC n. ${ }^{\circ} 163,15977-16008$.

DECRETO 237/2015, de 22 de diciembre, por el que se establece el currículo de Educación Infantil y se implanta en la Comunidad Autónoma del País Vasco. BOPV n. ${ }^{\circ}$ 9, 1-50.

DECRETO 25/2007, de 4 de mayo, por el que se establece el Currículo del Segundo Ciclo de Educación/Infantil en la Comunidad Autónoma de La Rioja. BOR n. ${ }^{\circ}$ 62, 3287-3292.

DECRETO 254/2008, de 1 de agosto, por el que se establece el currículo del Segundo Ciclo de la Educación Infantil en la Comunidad Autónoma de la Región de Murcia. BORM n. ${ }^{\circ}$ 182, 24960-24973.

DECRETO 330/2009, de 4 de junio, por el que se establece el currículo de la educación infantil en la Comunidad Autónoma de Galicia. DOG n. ${ }^{\circ} 121$, 10773-10799.

DeCRETO 38/2008, de 28 de marzo, del Consell, por el que se establece el currículo del segundo ciclo de la Educación Infantil en la Comunitat Valenciana. DOCV n. ${ }^{\circ} 5734,55018-55047$.

DECRETO/4/2008, de 11 de enero, por el que se aprueba el Currículo de Educación Infantil para la Comunidad Autónoma de Extremadura. DOE n. $12,1226-1272$.

DECRETO 67/2007, de 29-05-2007, por el que se establece y ordena el currículo del segundo ciclo de la Educación Infantil en la Comunidad Autónoma de Castilla-La Mancha. DOCM n. ${ }^{\circ} 116,14743-14759$.

DECRETO 71/2008, de 27 de junio, por el cual se establece el currículo de la educación infantil en las Islas Baleares. BOIB n. ${ }^{\circ}$ 92, 177-191. 
DECRETO 79/2008, de 14 de agosto por el que se establece el currículo del segundo ciclo de Educación infantil en la Comunidad Autónoma de Cantabria. BOC n. ${ }^{\circ}$ 164, 11543-11559.

DECRETO 85/2008, de 3 de septiembre, por el que se establece el currículo del segundo ciclo de Educación Infantil. BOPA n. ${ }^{\circ} 212,20440-20456$.

DECRETO ForAL 23/2007, de 19 de marzo, por el que se establece el currículo de las enseñanzas del segundo ciclo de la Educación Infantil de la Comunidad Foral de Navarra. BON n. ${ }^{\circ}$ 51, 9-42.

López Castro, Miguel (2004). Prólogo. En López Castro, Miguel (Coord.). Introducción al flamenco en el currículum escolar. Madrid: Akal-Universidad Internacional de Andalucía, (pp. 9-12).

ORDEN ECI /3960/2007, de 19 de diciembre, por la que se establece el currículo y se regula la ordenación de la educación infantil. Texto Consolidado. BOE n. ${ }^{\circ}$, 1-36.

ORDEN de 28 de marzo de 2008, del Departamento de Educación, Cultura y Deporte, por la que se aprueba el currículo de la Educación Infantil y se autoriza su aplicación en los centros docentes de la Comunidad Autónoma de Aragón. BOA n. ${ }^{\circ}$ 43, 4943-4974.

ORDEN de 5 de agosto de 2008, por la que se desarrolla el Currículo correspondiente a la Educación Infantil en Andalucía. BOJA n. ํ 169, 17-53.

PAdial-Ruz, Rosario, IbáÑEZ-Granados, Delia, FERnÁndez Hervás, Marina y UBAGo-JiMÉNEZ, José Luis (2019), Proyecto de baile flamenco: desarrollo motriz y emocional en educación infantil. Retos, 35, 396-401. https://doi.org/10.47197/retos.v0i35.63292

RuEDA JiMÉNEZ, Esperanza. (2004). Didáctica del flamenco en Educación Infantil. En Miguel López Castro (Coord.), Introducción al flamenco en el currículum escolar. Madrid: Akal.-Universidad Internacional de Andalucía, pp. 13-19.

SÁNCHEZ MARÍN, Calixto y NAVARRO GARCíA, José Luis (1998). Aproximación a una didáctica del flamenco. Sevilla: Junta de Andalucía (recuperado de http://flun.cica.es/flamcd/index.html).

UTRILLA ALMAGRO, Jerónimo (2007). El flamenco se aprende: teoría y didáctica de la enseñanza del flamenco. Córdoba: Ediciones Toro Mítico.

VALERO VARGAS, Paco (2004). El flamenco en el cole: experiencias prácticas. En Miguel López Castro (Coord.), Introducción al flamenco en el currículum escolar. Madrid: Akal.-Universidad Internacional de Andalucía, pp. 79-105. 